\title{
Evaluación de los efectos sinérgicos de cromo y plomo durante el proceso de fitorremediación con berro (Nasturtium officinale) en un humedal artificial
}

\author{
Evaluation of the synergistic effects of chromium and lead during the process of phytoremediation with
} watercress (Nasturtium officinale) in an artificial wetland

\begin{abstract}
Julia Mariana Márquez-Reyes*, Arcadio Valdés-González², Celestino García-Gómez'; Humberto Rodríguez-Fuentes, Julián Gamboa- Delgado ${ }^{3}$, Hugo Luna-Olvera ${ }^{4}$

Universidad Autónoma de Nuevo León, Facultad de Agronomía, Laboratorio de Remediación Ambiental. Av. Francisco Villa S/N, Colonia Ex Hacienda El Canadá, General Mariano Escobedo, Nuevo León, México. C.P. 66455.

2 Universidad Autónoma de Nuevo León, Facultad de Ciencias Biológicas, Laboratorio de Acuicultura. Av. Universidad S/N, Cd. Universitaria, San Nicolás de los Garza, Nuevo León, México. C.P. 66450.

3 Universidad Autónoma de Nuevo León, Facultad de Ciencias Biológicas, Departamento de Ecología. Av. Universidad S/N, Cd. Universitaria, San Nicolás de los Garza, Nuevo León, México. C.P. 66450.

${ }^{4}$ Universidad Autónoma de Nuevo León, Facultad de Ciencias Biológicas, Instituto de Biotecnología. Av. Universidad S/N, Cd. Universitaria, San Nicolás de los Garza, Nuevo León, México. C.P. 66450.
\end{abstract}

\section{RESUMEN}

La contaminación por metales pesados ha aumentado con los años debido a sus diferentes aplicaciones. Se han evaluado distintas especies vegetales para bioacumular metales pesados, el berro es una especie con capacidad de fitorremediación. Sin embargo, falta información sobre la sinergia que ocurre cuando dos metales en el medio están presentes y condicionan su acumulación en las plantas. El objetivo de esta investigación fue evaluar el efecto sinérgico en la acumulación simultánea de $\mathrm{Pb}$ y $\mathrm{Cr}(\mathrm{VI})$ en berro. Se utilizó un sistema cerrado y un humedal artificial para evaluar el comportamiento de los metales en presencia de berro, cuantificando al final de cada experimento la concentración acumulada en tallos, hoja y raíz. La mayor concentración de ambos metales fue en raíz $(\mathrm{Pb}>\mathrm{Cr}$ ). Al incrementarse la presencia de $\mathrm{Cr}(\mathrm{VI})$ en solución, la planta absorbe más metal, y en combinación con $\mathrm{Pb}$ el índice de tolerancia se aumenta y el factor de translocación disminuye. Dentro del humedal construido el porcentaje de remoción de $\mathrm{Pb}$ y $\mathrm{Cr}$ total fue del >99.9\% (100 $\left.\mathrm{mg} \mathrm{L}^{-1}\right)$ y $95 \%$ (28.5 $\left.\mathrm{mg} \mathrm{L}^{-1}\right)$, respectivamente. Los resultados obtenidos indican que existen interacciones ambientales, físicas y químicas que determinan la capacidad de bioacumulación en el berro, de los metales evaluados.

Palabras clave: Rizofiltración, Humedal artificial, Índice de tolerancia, Factor de bioconcentración, Factor de Traslocación.

\section{ABSTRACT}

Heavy metal pollution has increased over the years due to its different applications. Different plant species have been evaluated to bioaccumulate heavy metals; watercress is a species with phytoremediation capacity. However, there is little information on the synergy that occurs when two metals in the medium are present and condition their accumulation in plants. The objective of this research was to

*Autor para correspondencia: Julia Mariana Márquez-Reyes

Correo electrónico: jmmarquez23@gmail.com

Recibido: 19 de noviembre de 2019

Aceptado: 19 de febrero de 2020 evaluate the synergistic effect on the simultaneous accumulation of $\mathrm{Pb}$ and $\mathrm{Cr}(\mathrm{VI})$ in watercress. We used a batch system and an artificial wetland to evaluate the behavior of metals in the presence of watercress, quantifying at the end of each experiment the cumulative concentration in stems, leaf, and root. The highest concentration of both metals occurred in the root section $(\mathrm{Pb}>\mathrm{Cr}$ ). As the presence of $\mathrm{Cr}(\mathrm{VI})$ in solution increases, the plant absorbs more metal, and combination with $\mathrm{Pb}$ the tolerance index is increased and the translocation factor decreases. Within the constructed wetland the percentage of total $\mathrm{Pb}$ and $\mathrm{Cr}$ removal was $>99.9 \%$ (100 mg $\left.\mathrm{L}^{-1}\right)$ and $95 \%$ (28.5 $\left.\mathrm{mg} \mathrm{L}^{-1}\right)$ respectively. The results show the presence of effects of environmental, physical and chemical interactions that determine the capacity of bioaccumulation of the metals evaluated in watercress.

Key Words: Rhizofilter, Constructed wetland, Tolerance index, Bioconcentration factor, Translocation factor.

\section{INTRODUCCIÓN}

Los metales pesados son productos generados a partir de diferentes actividades antropogénicas que con el tiempo han aumentado sus usos y su acumulación, causando diferentes tipos de problemas en los ecosistemas. Existen metales pesados como el $\mathrm{Pb}$ y $\mathrm{Cr}$ (VI) que no tienen utilidad biológica y que además son tóxicos y no biodegradables (Adriano 2003; Yadav et al., 2012; Maceda-Veiga et al., 2013). Al ambiente se liberan 91,000 toneladas métricas de $\mathrm{Cr}(\mathrm{VI})$ por año en agua, aire y suelo, donde su concentración puede variar en agua dulce $\left(0.1\right.$ a $\left.117 \mu \mathrm{g} \mathrm{L}^{-1}\right)$, agua marina (0.2 a 0.5 $\mu \mathrm{L} \mathrm{L}^{-1}$ ) y suelo (5 a $1000 \mathrm{mg} \mathrm{kg}^{-1}$ ) (Pawlisz et al., 1997; ATSDR, 2012). Mientras que las concentraciones de $\mathrm{Pb}$, por emisiones naturales alcanzan 19000 ton/año debido a los desgastes geológicos y emisiones volcánicas y por actividades antropogénicas se estiman 120000 ton/año (Lima et al., 2005). El $\mathrm{Pb}$ en el ecosistema puede estar presente en agua para beber 
(15 $\left.\mathrm{mg} \mathrm{L}^{-1}\right)$, agua marina ( 2 a $\left.30 \mathrm{pg} \mathrm{L}^{-1}\right)$, agua de ríos ( 3 a 30 $\left.\mathrm{ng} \mathrm{L^{-1 }}\right)$, fitoplancton (5 a $\left.10 \mathrm{mg} \mathrm{L}^{-1}\right)$, peces de agua dulce $(0.5$ a $\left.1000 \mathrm{ng} \mathrm{L}^{-1}\right)$, ostras $\left(500 \mathrm{ng} \mathrm{L}^{-1}\right)$, sedimentos $\left(500 \mathrm{mg} \mathrm{L}^{-1}\right)$, suelo (30 a $300 \mathrm{mg} \mathrm{kg}^{-1}$ ) y aire $\left(0.5\right.$ a $0.1 \mu \mathrm{g} \mathrm{m}^{-3}$ ) (Frank et al., 2019; EPA, 2018). La interacción química del metal en suelo y agua está regulada por la especiación química del mismo, la toxicidad, la concentración, el pH, el contenido de materia orgánica y la capacidad de intercambio catiónico que en algunos escenarios producen biomagnificación (Devallois et al., 2008; Yadav et al., 2010; Li et al., 2017).

El rápido crecimiento, tasa alta de generación de biomasa y capacidad de acumulación de contaminantes en partes cosechables son algunas de las características más deseables que tienen las plantas empleadas en biorremediación (Seth, 2011). La bioacumulación de metales pesados en plantas ha sido evaluada ampliamente (Marchand et al., 2010), pero debido a la gran diversidad que existe, aún faltan muchas especies por estudiar. El berro (Nasturtium officinale) es una planta fácil de cultivar en ambientes acuáticos y terrestres, siendo considerado como una especie con alta actividad antioxidante, antiinflamatoria, antialérgenica, cardioprotectora, antidiabética y de gran utilidad en el sector cosmetológico y ambiental (Rose et al., 2005; Klimek-Szczykutowicz et al., 2018). En estudios de biorremediación se ha demostrado su capacidad acumuladora de metales $(\mathrm{Cr}, \mathrm{Cu}, \mathrm{Cd}, \mathrm{Co}, \mathrm{Zn}$ y $\mathrm{Ni}$ ), metaloides (As) y compuestos radiactivos (Zurayk et al., 2001a; Zurayk et al., 2001 b; Aslan et al., 2003; Kara, 2005; Saygideger y Dogan, 2005; Duman et al., 2009; Ozturk et al., 2010; Cordeiro et al., 2016). Lin et al. (2015) evaluaron el berro para acumular $\mathrm{Cd}$ a diferentes concentraciones, encontrando que el contenido del metal aumentó en la planta al incrementarse la concentración en la solución. Las raíces y tallos del berro pueden acumular más $\mathrm{Cd}$ cuando están en combinación con otras especies vegetales y sustratos sólidos (Huang et al., 2017; Li et al., 2017).

Para determinar la capacidad de remediación que tiene una planta es necesario estimar los factores de bioconcentración, traslocación e índice de tolerancia. Estos indican el lugar y la capacidad de acumulación de contaminantes en la planta; así como su nivel de tolerancia a los contaminantes (Wang et al., 2019). De acuerdo a los factores e índices determinados, las especies vegetales pueden clasificarse como bioacumuladoras e hiperacumuladoras. Existen pocos estudios en la literatura que evalúen la sinergia entre metales pesados y los factores de bioconcentración, traslocación e índice de tolerancia (Ng et al., 2016; Favas et al., 2018; Vidal del Campos et al., 2019).

Los objetivos del presente trabajo fueron: (1) evaluar la acumulación de $\mathrm{Pb}$ y $\mathrm{Cr}(\mathrm{VI})$ en berro; (2) evaluar la sinergia de acumulación entre $\mathrm{Pb}$ y $\mathrm{Cr}(\mathrm{VI})$ en berro estimando el factor de bioconcentración, traslocación e índice de tolerancia y (3) evaluar en un humedal construido alimentado con agua sintética, el porcentaje de remoción de $\mathrm{Pb}$ y $\mathrm{Cr}(\mathrm{VI})$.

\section{MATERIALES Y MÉTODOS Efecto de $\mathrm{Pb}$ y $\mathrm{Cr}$ (VI)}

La solución nutritiva de trabajo (L-C) estaba compuesta por $\mathrm{KNO}_{3}\left(101.0 \mathrm{~g} \mathrm{~L}^{-1}\right) ; \mathrm{KH}_{2} \mathrm{PO}_{4}\left(27.2 \mathrm{~g} \mathrm{~L}^{-1}\right) ; \mathrm{MgSO}_{4} \cdot 7 \mathrm{H}_{2} \mathrm{O}(98.6$ $\left.\mathrm{g} \mathrm{L}^{-1}\right) ; \mathrm{Ca}\left(\mathrm{NO}_{3}\right)_{2} \cdot 4 \mathrm{H}_{2} \mathrm{O}\left(295.0 \mathrm{~g} \mathrm{~L}^{-1}\right) ; \mathrm{NH}_{4} \mathrm{Cl}\left(1.0 \mathrm{~g} \mathrm{~L}^{-1}\right) ; \mathrm{Na}_{2} \mathrm{SO}_{4}(4.5$ $\left.\mathrm{g} \mathrm{L}^{-1}\right) ; \mathrm{CaCl}_{2}\left(0.06 \mathrm{~g} \mathrm{~L}^{-1}\right) ; \mathrm{FeSO}_{4} \cdot 7 \mathrm{H}_{2} \mathrm{O}\left(1.3 \mathrm{~g} \mathrm{~L}^{-1}\right) ; \mathrm{ZnSO}_{4} \cdot 7 \mathrm{H}_{2} \mathrm{O}(0.2$ $\left.\mathrm{g} \mathrm{L}^{-1}\right) ; \mathrm{CuSO}_{4} \cdot 7 \mathrm{H}_{2} \mathrm{O}\left(0.08 \mathrm{~g} \mathrm{~L}^{-1}\right)$ a pH $5 \pm 0.2$. Para los experimentos monometálicos se utilizaron recipientes de plástico de $1 \mathrm{~L}$ con solución nutritiva L-C y tres plantas previamente pesadas (10 cm de longitud) se adicionaron concentraciones conocidas $\left(3,6,12,15\right.$ y $\left.20 \mathrm{mg} \mathrm{L}^{-1}\right)$ de $\mathrm{Pb}$ y $\mathrm{Cr}(\mathrm{VI})$, los recipientes fueron colocados dentro de una cámara climática (Lab-Line Instruments, INC, biotonette Mark III) con una iluminación de $270 \mu \mathrm{mol} \mathrm{m} \mathrm{m}^{-2} \mathrm{~s}^{-2}$, un fotoperíodo de luz: oscuridad $16 \mathrm{~h}: 8 \mathrm{~h}$ y $25 \pm 3^{\circ} \mathrm{C}$ durante 18 y 20 días, respectivamente.

Para evaluar la sinergia entre metales se establecieron las mismas condiciones de iluminación y fotoperiodo, al igual que el número de plantas por recipiente durante 15 días. Los tratamientos mezcla fueron: $\mathrm{Pb} 5 \mathrm{mg} \mathrm{L}^{-1}-\mathrm{Cr}(\mathrm{VI}) 5 \mathrm{mg} \mathrm{L}^{-1}, \mathrm{~Pb}$ $10 \mathrm{mg} \mathrm{L}^{-1}-\mathrm{Cr}(\mathrm{VI}) 5 \mathrm{mg} \mathrm{L}^{-1}$ y Pb $5 \mathrm{mg} \mathrm{L}^{-1}-\mathrm{Cr}(\mathrm{Vl}) 10 \mathrm{mg} \mathrm{L}^{-1}$. Las soluciones nutritivas se reemplazaron completamente cada 4 días para evitar la fluctuación de las concentraciones debido a evaporación y evapotranspiración.

Después del período experimental, las plantas se retiraron y enjuagaron con agua destilada. Todo el material vegetal fue secado a $50^{\circ} \mathrm{C}$ durante $30 \mathrm{~h}$. Para la cuantificación del metal en la planta de todos los experimentos, se tomaron $200 \mathrm{mg}$ de biomasa seca (raíces, brotes y hojas), se le adicionaron $15 \mathrm{~mL}$ de una mezcla de $\mathrm{H}_{2} \mathrm{O}_{2} / \mathrm{HNO}_{3}(3: 10 \mathrm{v} / \mathrm{v})$ y se mantuvo a $85^{\circ} \mathrm{C}$ durante $10 \mathrm{~min}$. Posteriormente, el material fue filtrado y analizado por espectrometría de absorción atómica (EAA, GBC Mod. 932AA); se utilizó una lámpara de cátodo hueco específica para $\mathrm{Pb}(217 \mathrm{~nm})$ y $\mathrm{Cr}(357.9 \mathrm{~nm})$, una lámpara de deuterio para la corrección de fondo y un flujo de la mezcla de aire-acetileno para $\mathrm{Pb}(13.50$ - $1.60 \mathrm{~L}$ $\left.\mathrm{min}^{-1}\right)$ y $\mathrm{Cr}\left(13.50-3.05 \mathrm{~L} \mathrm{~min}^{-1}\right)$ diferente.

\section{Índice de tolerancia, factor de bioconcentración y traslo- cación}

El índice de tolerancia (IT) se estimó considerando la biomasa vegetal expuesta al metal $\mathrm{B}_{t}$ ( $\mathrm{g}$ planta) y la biomasa vegetal control $B_{c}$ (g planta), y se calculó empleando la ecuación (1). A valores mayores de IT, mayor tolerancia tiene la planta al metal (Shi et al., 2011).

$$
I T={ }^{B}{ } B_{C} \quad \text { Ecuación (1) }
$$

El factor de bioconcentración (FBC) indica la relación entre la concentración ( $\left.\mathrm{mg} \mathrm{kg}^{-1}\right)$ del metal en los tallos $\left(C_{\text {tallos }}\right)$ y la concentración $\left(\mathrm{mg} \mathrm{kg}^{-1}\right)$ del metal en la solución $\left(\mathrm{C}_{\text {solución }}\right)$. El FBC evalúa la capacidad (ecuación 2) que tiene la planta para absorber el metal de la solución y trasladarlo a los tejidos superiores (Liu et al., 2008).

$$
F B C=C_{\text {tallos }} / C_{\text {solución }} \quad \text { Ecuación (2) }
$$


Mientras que el factor de translocación (FT), proporciona una estimación de la capacidad que tiene la planta en transportar el metal entre las raíces y los tallos (Ribelro De Souza et al., 2012). Se calcula con la ecuación (3), en donde $\mathrm{A}_{\text {tallos }}\left(\mathrm{mg} \mathrm{kg}^{-1}\right)$ es la concentración del metal total acumulado en brotes y $A_{\text {raices }}\left(\mathrm{mg} \mathrm{kg}^{-1}\right)$ la concentración del metal acumulado en raíces.

$$
F T=A_{\text {tallos }} / A_{\text {raíces }} \quad \text { Ecuación (3) }
$$

\section{Humedal construido (HCs)}

Para simular las condiciones naturales del crecimiento del berro, se utilizó un tanque rectangular con dimensiones de $85 \times 44 \times 13 \mathrm{~cm}$ (largo $x$ ancho $x$ alto), con una pendiente del $2 \%$ y grava como sustrato $(5 \mathrm{~cm}$ de profundidad). La porosidad establecida dentro del HCs fue de $24 \%$ y con ayuda de una bomba peristáltica se alimentó el influente manteniendo un tiempo de residencia hidráulico de 2 días con 24 min. Las condiciones ambientales fueron las mismas ya reportadas en la sección anterior. Dentro del HCs se sembraron 50 plantas de berro de aproximadamente $8 \mathrm{~cm}$ de longitud y se estableció una fase de acondicionamiento por 12 días, los primeros 10 días se hizo circular solución nutritiva L-C y los restantes 2 días se agregó $\mathrm{Pb}$ y $\mathrm{Cr}(\mathrm{VI})$ a la solución $100 \mathrm{mg} \mathrm{L}^{-1}$ y $30 \mathrm{mg} \mathrm{L}^{-1}$, respectivamente; esto último con la finalidad de minimizar la interacción de los metales con el sustrato rocoso y evaluar el comportamiento del berro a concentraciones más elevadas a las evaluadas en las etapas experimentales previas. Posteriormente se renovó la solución y se mantuvo el experimento por 11 días. Se tomaron plantas $(n=12)$ a distintos tiempos de operación del humedal ( 2 y 11 días), las cuales fueron tratadas como se mencionó anteriormente para cuantificar los metales pesados en raíces, tallos y hojas. Diariamente se tomaron muestras del efluente para determinar el pH (APHA, 2005) y la concentración total de metales por EAA (GBC Mod. $932 \mathrm{AA})$.

\section{Balance de masa y especiación de $\mathrm{Pb}$ y $\mathrm{Cr}$}

Se estableció un control para evaluar las interacciones del metal con la grava, colocando $200 \mathrm{~mL}$ de solución a 100 $\mathrm{mg} \mathrm{L}^{-1}$ y $30 \mathrm{mg} \mathrm{L}^{-1}$ de $\mathrm{Pb}$ y $\mathrm{Cr}(\mathrm{VI})$, respectivamente con 200 $\mathrm{g}$ de grava. Se dejaron interactuar por $48 \mathrm{~h}$ y se tomó una alícuota para cuantificar la concentración del metal en la solución y por diferencia conocer la concentración que dejó de estar soluble. La ecuación general de balance de masa establecida en el sistema considera la concentración inicial de cada metal en el influente, la concentración total de metal en el efluente, la concentración retenida en el berro y la grava y la concentración remanente no cuantificada en el estudio (ecuación 4).

$$
M e_{\text {influente }}=M e_{\text {efluente }}+M e_{\text {berro }}+M e_{\text {grava }}+M e_{\text {no cuantificacdo }} \text { Ecuación (4) }
$$

Donde $M_{e}$ es la concentración del metal pesado cuantificado en diferentes partes del humedal artificial (influente, efluente, berro y grava) o el remanente no cuantificado.
Para evaluar la especiación química de los metales se utilizó el software Visual MINTEQ 3.0. Se consideraron dos escenarios; los metales en presencia de grava y los metales en ausencia de grava, para ambos escenarios se mantuvieron las mismas características químicas de la solución, fuerza iónica, $\mathrm{pH}$ y temperatura.

\section{Análisis estadístico}

Todos los experimentos se realizaron por triplicado en las distintas fases experimentales. Los datos obtenidos fueron sometidos a un análisis de varianza de una vía y una prueba de comparación de medias (Tukey) cuando se encontró significancia entre los tratamientos $(P<0.05)$. Se utilizó el paquete estadístico SPSS 17.0 (Inc., EE. UU.) y OriginPro 6.0.

\section{RESULTADOS Y DISCUSIÓN \\ Acumulación de metales en berro}

En las fracciones analizadas de berro se encontraron diferencias estadísticas entre las concentraciones cuantificadas en los tratamientos. La mayor acumulación de $\mathrm{Pb}$ en hojas, tallos y raíces fue en los tratamientos de 15, 12 y $3 \mathrm{mg}$ $\mathrm{L}^{-1}$, con valores $2,0.97$ y $31 \mathrm{~g} \mathrm{~kg}^{-1}$, respectivamente. De acuerdo a los resultados obtenidos el berro tiene la capacidad de acumular $\mathrm{Pb}$ en raíces $>$ hojas $>$ tallos (Fig. 1). Keser y Saygideger (2010), reportaron el mismo comportamiento de acumulación y cuantificación similar en hojas y tallos de berro. En los resultados obtenidos no se observó una correlación directa entre la concentración de $\mathrm{Pb}$ acumulada en berro y la concentración de $\mathrm{Pb}$ en solución. Esto difiere con los reportado por Lin et al. (2015), cuando evaluaron la acumulación de $\mathrm{Cd}$ en berro, mostrando que a mayor concentración del metal mayor acumulación en la planta. Lo anterior puede deberse a las interacciones químicas que se puedan dar en la solución que limiten en algunas situaciones la disponibilidad del $\mathrm{Pb}$ hacia el berro. Sin embargo, para $\mathrm{Cr}(\mathrm{VI})$ se observó que conforme aumenta la concentración de metal en la solución, aumenta la acumulación en raíces $<$ tallos $<$ hojas (Fig. 1). Duman et al. (2009), reportaron resultados similares al presente al cuantificar $\mathrm{Cr}$ en raíces > tallos> hojas de berro. De igual manera en plantas de trigo, avena y sorgo la mayor acumulación de $\mathrm{Cr}$ fue en raíces (López-Luna et al., 2009). De acuerdo a los resultados obtenidos, el berro presenta distintas afinidades entre metales y se clasifica como rizofiltradora. Huang et al. (2017), la clasifica de dicha manera por acumular metales en raíces y Li et al. (2017), la considera una especie hiperacumuladora de cadmio.

\section{Sinergia en la acumulación de metales}

Entre los distintos tratamientos evaluados se encontraron diferencias estadísticas $(P \leq 0.05)$ en la acumulación de ambos metales en las distintas fracciones del berro analizado. El berro acumuló más $\mathrm{Pb}$ en hojas y raíces cuando ambos metales se encontraban a la misma concentración. Mientras en los tallos la mayor cuantificación ocurrió en la concentración más alta de $\mathrm{Pb}$. Se obtuvo el mismo comportamiento observado en el experimento monometálico, la mayor 

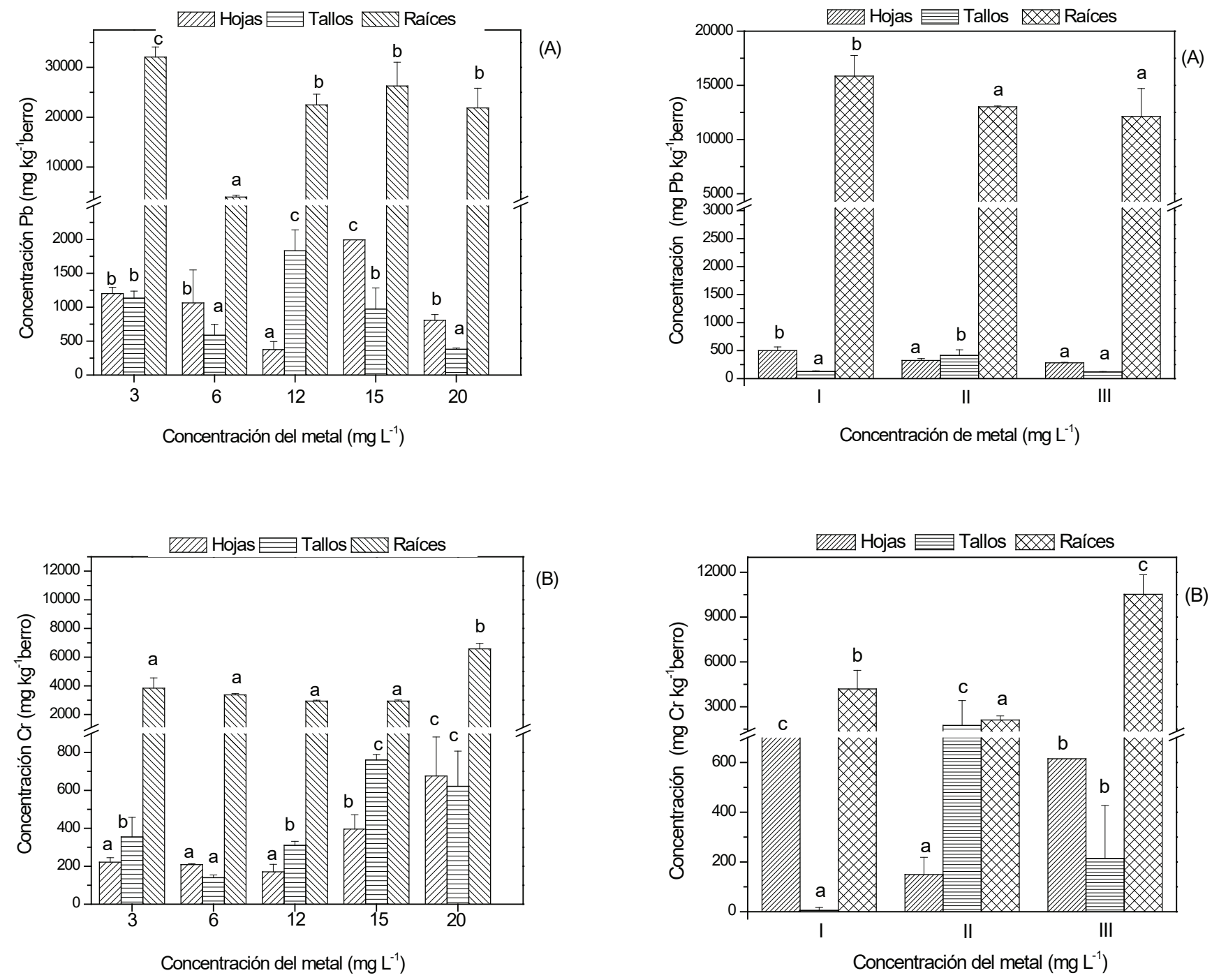

Figura 1. Cuantificación de plomo (A) y cromo (B) en hojas, tallos y raíces de berro expuesto a distintas concentraciones del metal. Los datos son promedio de tres replicas con su respectiva desviación estándar. Las diferentes letras $a, b, c$ indican diferencias significativas $(P \leq 0.05)$.

Figure 1. Lead (A) and chromium (B) quantification in leaves, stems and roots of watercress exposed to different concentrations of the metal. The data is an average of three replicas with their respective standard deviation. Different letters $a, b, c$ indicates significant differences $(P \leq 0.05)$.

acumulación de $\mathrm{Pb}$ fue raíces $>$ hojas $>$ tallos. La presencia de $\mathrm{Cr}$ en hojas ocurrió cuando ambos metales tenían la misma concentración. Mientras que la mayor cuantificación de $\mathrm{Cr}$ en tallos fue en la mayor concentración de $\mathrm{Pb}$. En las raíces se acumula más $\mathrm{Cr}$ cuando la concentración del mismo es mayor. Una mayor concentración de $\mathrm{Pb}$ favorece la acumulación de $\mathrm{Cr}$ en tallos. Lin et al. (2015), reportaron que la mayor concentración de $\mathrm{Cd}$ en berro se acumuló en raíces < tallos. Está mayor afinidad a acumular $\mathrm{Cr}$ se puede deber a que la planta expresa mecanismos metabólicos que contrarrestan el efecto tóxico del metal por acción enzimática o actividad bacteriana, modificando la forma química de $\mathrm{Cr}$ (VI) a $\mathrm{Cr}$ (III),

Figura 2. Cuantificación de plomo (A) y cromo (B) en hojas, tallos y raíces de berro expuesto a (I): Pb $5 \mathrm{mg} \mathrm{L}^{-1}-\mathrm{Cr} 5 \mathrm{mg} \mathrm{L}^{-1}$, (II): Pb $10 \mathrm{mg} \mathrm{L}^{-1}-\mathrm{Cr} 5 \mathrm{mg}$ $\mathrm{L}^{-1}$ y (III): $\mathrm{Pb} 5 \mathrm{mg} \mathrm{L}^{-1}-\mathrm{Cr} 10 \mathrm{mg} \mathrm{L}^{-1}$. Los datos son promedio de tres replicas con su respectiva desviación estándar. Las diferentes letras $\mathrm{a}, \mathrm{b}, \mathrm{c}$ indican diferencias significativas $(P \leq 0.05)$.

Figure 2. Lead (A) and chromium (B) quantification in leaves, stems and roots of watercress exposed to (I): $\mathrm{Pb} 5 \mathrm{mg} \mathrm{L}^{-1}-\mathrm{Cr} 5 \mathrm{mg} \mathrm{L}^{-1}$, (II): Pb $10 \mathrm{mg}$ $\mathrm{L}^{-1}-\mathrm{Cr} 5 \mathrm{mg} \mathrm{L}^{-1}$ and (III): $\mathrm{Pb} 5 \mathrm{mg} \mathrm{L}^{-1}-\mathrm{Cr} 10 \mathrm{mg} \mathrm{L}^{-1}$. The data is an average of three replicas with their respective standard deviation. Different letters $a, b$, $c$ indicates significant differences $(P \leq 0.05)$.

transformándolo en una forma química asimilable para la planta y no tóxica (Jin et al., 2017).

Las interacciones físicas, químicas y biológicas entre $\mathrm{Pb}$ y $\mathrm{Cr}(\mathrm{VI})$, resultan en una disminución general de la capacidad del berro en acumular los metales. Las concentraciones promedio cuantificadas en la planta en presencia de ambos metales indican una disminución en la acumulación de $\mathrm{Pb}$ del 56,85 y $20 \%$ en hojas, tallos y raíces, respectivamente. Mientras que, para $\mathrm{Cr}$ total la capacidad disminuyó 73 y 76 $\%$ en tallos y raíces, respectivamente. La concentración de $\mathrm{Cr}$ total en hojas aumentó $128 \%$, a mayor concentración de $\mathrm{Cr}$ 
(VI) en la mezcla, este comportamiento se presenta probablemente por una sobre expresión de genes que codifican para transportadores de membranas que incrementan el translocamiento de los metales a las vacuolas como medida de detoxificación (Rascio y Navari-Izzo, 2011).

\section{Índice de tolerancia, factor de bioconcentración y factor de translocación}

El índice de translocación y el factor de bioconcentración cuantificados en ambos metales fueron disminuyendo conforme se incrementó la concentración del metal en la solución (Tabla 1). A pesar de su alta toxicidad para la mayoría de las especies vegetales, algunas plantas tienen la capacidad de absorber, acumular y tolerar altas concentraciones de metal, la acumulación es generalmente proporcional a la concentración del elemento en el medio (Jasrotia et al., 2017), donde valores $>1000$ de FBC $y>1$ FT las clasifica como plantas hiperacumuladoras (Islam et al., 2015). Por lo anterior, el berro es clasificado con baja capacidad para acumular altas concentraciones de $\mathrm{Pb}$ y $\mathrm{Cr}(\mathrm{VI})$ en sus órganos subterráneos, resultados similares están reportados para Plantago major $L$., Fontinalis squamosa, Brachythecium rivulare, Platyhypnidium riparioides, Thamnobryum alopecurum, Lemanea fluviatilis y Pistia stratiotis (Galal y Shehata, 2015; Favas et al., 2018; Vidal de Campos et al., 2019).

El FT en presencia de $\mathrm{Pb}$ fue mayor en concentraciones bajas y disminuyó al incrementarse la concentración. A altas concentraciones de $\mathrm{Cr}(\mathrm{VI})$ en la solución, mayor $\mathrm{FT}$, este comportamiento puede ser consecuencia del daño de la membrana plasmática, que interfiere con la funcionalidad de los transportadores e impide los mecanismos normales de captación celular (Shri et al., 2009). Los valores obtenidos de $\mathrm{FT}$, indican una baja eficacia de trasladar el metal en raíces a partes aéreas. Duman y Ozturk (2010), trabajaron con Nastur- tium officinale exponiéndolo a níquel obteniendo resultados similares a los encontrados en este estudio. En otros estudios se ha observado el mismo comportamiento tal es el caso de Pistia stratiotis (Das et al., 2014), Eichhornia crassipes (Souza et al., 2018), Elodea canadensis (Picco et al., 2019) Typha latifolia y Juncus effusus (Favas et al., 2012). Shi et al. (2011), sugieren que plantas con características similares a las encontradas en el presente estudio sean consideradas especies estabilizadoras de metales pesados o rizofiltradoras (Olmos-Márquez et al., 2012).

Para las mezclas metálicas el IT y FBC en Pb, fue mayor a concentraciones superiores de $\mathrm{Cr}(\mathrm{VI})$ en solución. El valor mayor de FT se estimó cuando ambos metales se encontraron a la misma concentración (Tabla 1). El IT evaluado para $\mathrm{Cr}(\mathrm{VI})$, fue menor a altas concentraciones de $\mathrm{Pb}$, los demás tratamientos no mostraron diferencias significativas. Mientras el $\mathrm{FBC}$ para $\mathrm{Cr}(\mathrm{VI})$ se incrementó a mayor concentración de $\mathrm{Pb}$ y el FT no mostró diferencias significativas entre los tratamientos. Los resultados obtenidos de las mezclas de metales indican que ambos metales a concentraciones similares favorecen la translocación de $\mathrm{Pb}$; cuando alguno de los metales se encuentra en mayor concentración en la solución, se promueve la acumulación del otro. Los valores obtenidos de IT y FBC en las mezclas se pueden comparar en magnitud a los obtenidos individualmente en los tratamientos de 3 y 6 $\mathrm{mg} \mathrm{L}^{-1}$ de cada metal. Mientras que, el FT es menor en mezcla en comparación con lo cuantificado por metal individual. Lo anterior indica que existen interacciones químicas entre metales que pueden modificar la toxicidad, la disponibilidad y su acumulación en berro. Se sugiere para futuros trabajos evaluar un mayor número de variables dentro del sistema para establecer las interacciones que puede favorecer la bioacumulación de algún metal en particular.

Tabla 1. Índice de tolerancia, factor de bioconcentración y factor translocación de berro en presencia de plomo, cromo y mezclas metálicas.

Table 1. Tolerance index, bioconcentration factor and translocation factor the watercress in presence of lead, chromium and metal mixtures.

\begin{tabular}{|c|c|c|c|c|c|c|}
\hline $\begin{array}{l}\text { Concentración } \\
\left(\mathrm{mg} \mathrm{L}^{-1}\right)\end{array}$ & & $\mathbf{P b}$ & & & $\mathrm{Cr}$ & \\
\hline Metal & IT & FBC $\left(\mathrm{mg} \mathrm{kg}^{-1}\right)$ & FT $\left(\mathrm{mg} \mathrm{kg}^{-1}\right)$ & IT & $\mathrm{FBC}\left(\mathrm{mg} \mathrm{kg}^{-1}\right)$ & FT $\left(\mathrm{mg} \mathrm{kg}^{-1}\right)$ \\
\hline 3 & $0.39 \pm 0.01^{d}$ & $0.71 \pm 0.1^{d}$ & $0.63 \pm 0.01^{a}$ & $0.18 \pm 0.01^{c}$ & $0.34 \pm 0.1^{d}$ & $0.19 \pm 0.06^{\mathrm{a}}$ \\
\hline 6 & $0.25 \pm 0.02^{c}$ & $0.48 \pm 0.2^{c}$ & $0.15 \pm 0.01^{\mathrm{b}}$ & $0.16 \pm 0.08^{c}$ & $0.23 \pm 0.01^{c}$ & $0.41 \pm 0.03^{b}$ \\
\hline 12 & $0.21 \pm 0.01^{b}$ & $0.17 \pm 0.01^{b}$ & $0.09 \pm 0.03^{a}$ & $0.13 \pm 0.01^{b}$ & $0.17 \pm 0.01^{b}$ & $0.40 \pm 0.07^{b}$ \\
\hline 15 & $0.20 \pm 0.01^{b}$ & $0.26 \pm 0.02^{b}$ & $0.06 \pm 0.02^{\mathrm{a}}$ & $0.11 \pm 0.02^{b}$ & $0.10 \pm 0.01^{\mathrm{a}}$ & $0.55 \pm 0.01^{c}$ \\
\hline 20 & $0.02 \pm 0.01^{\mathrm{a}}$ & $0.09 \pm 0.01^{\mathrm{a}}$ & $0.08 \pm 0.005^{\mathrm{a}}$ & $0.04 \pm 0.001^{a}$ & $0.10 \pm 0.01^{\mathrm{a}}$ & $0.53 \pm 0.03^{c}$ \\
\hline \multicolumn{7}{|l|}{ Mezcla Pb-Cr(VI) } \\
\hline $5-5$ & $0.32 \pm 0.02^{b}$ & $0.17 \pm 0.1^{b}$ & $0.23 \pm 0.01^{b}$ & $0.30 \pm 0.02^{b}$ & $0.10 \pm 0.06^{a}$ & $0.03 \pm 0.01^{\mathrm{a}}$ \\
\hline $10-5$ & $0.26 \pm 0.03^{\mathrm{a}}$ & $0.03 \pm 0.001^{a}$ & $0.09 \pm 0.02^{\mathrm{a}}$ & $0.27 \pm 0.03^{\mathrm{a}}$ & $0.43 \pm 0.01^{b}$ & $0.03 \pm 0.01^{\mathrm{a}}$ \\
\hline $5-10$ & $0.37 \pm 0.03^{c}$ & $0.61 \pm 0.02^{c}$ & $0.06 \pm 0007^{a}$ & $0.31 \pm 0.03^{b}$ & $0.08 \pm 0.007^{\mathrm{a}}$ & $0.04 \pm 0.01^{\mathrm{a}}$ \\
\hline
\end{tabular}

Los valores representan la media y la desviación estándar para tres muestras independientes.

Diferentes letras en una misma columna indican diferencias significativas $(P \leq 0.05)$.

Values represent the mean and standard deviation from three independent samples.

Different letters in the same column indicate significant differences $(P \leq 0.05)$. 


\section{Humedal construido}

Durante la operación del HCs solo fue posible cuantificar la concentración de $\mathrm{Pb}\left(5.6 \mathrm{mg} \mathrm{L}^{-1}\right)$ en el efluente las primeras $48 \mathrm{~h}$ ya que posteriormente la concentración se encontraba por debajo del límite de cuantificación $(0.5 \mathrm{mg}$ $\left.\mathrm{L}^{-1}\right)$ del equipo de EAA. Este comportamiento se pudo deber a la interacción del $\mathrm{Pb}$ con la grava, ya que está compuesta por caliza, granito, dolomita, arenisca, cuarcita y minerales de cuarzo, los cuales reaccionan para formar hidróxidos metálicos y modificar el pH del medio (Naiya et al., 2009), promoviendo la precipitación del Pb. Dentro del HCs el pH se incrementó a 5.6, precipitándose el metal y haciéndose menos disponible para la planta. La cuantificación de $\mathrm{Pb}$ en berro al principio y final del experimento $\left(330 \mathrm{mg} \mathrm{kg}^{-1}\right)$ no indicó diferencias estadísticas.

Sin embargo, se cuantificaron $3.4 \mathrm{mg} \mathrm{L}^{-}$de $\mathrm{Cr}$ total en el efluente los primeros cuatro días y posteriormente $1.3 \mathrm{mg}$ $\mathrm{L}^{-1}$ (Figura 3). Al día 2 la cuantificación de $\mathrm{Cr}$ total en berro fue de $360 \mathrm{mg} \mathrm{kg}^{-1}$ y $606 \mathrm{mg} \mathrm{kg}^{-1}$ para el final del experimento. La acumulación de $\mathrm{Cr}$ en berro fue incrementándose a lo largo del tiempo. Esto se relaciona con los resultados obtenidos en la sección anterior, donde probablemente la toxicidad del $\mathrm{Cr}(\mathrm{VI})$ fue modificada, debido a una reducción química a $\mathrm{Cr}$ (III) y a la interacción superficial con la grava la cual modifica el pH del medio. Además, no se descarta la generación de exudados radiculares como agentes reductores o quelantes (Hellerich et al., 2008; Ovecka y Takac, 2014).

El humedal diseñado en este estudio logró remover 95 y $>99.9 \%$ de $\mathrm{Cr}$ total y $\mathrm{Pb}$, respectivamente. Los resultados observados indican que la acumulación de los metales depende de su disponibilidad hacia el área radicular y que esta puede modificarse por variables ambientales como $\mathrm{pH}$, temperatura, potencial óxido - reducción, concentración de iones, entre otros parámetros (Liu et al., 2019).

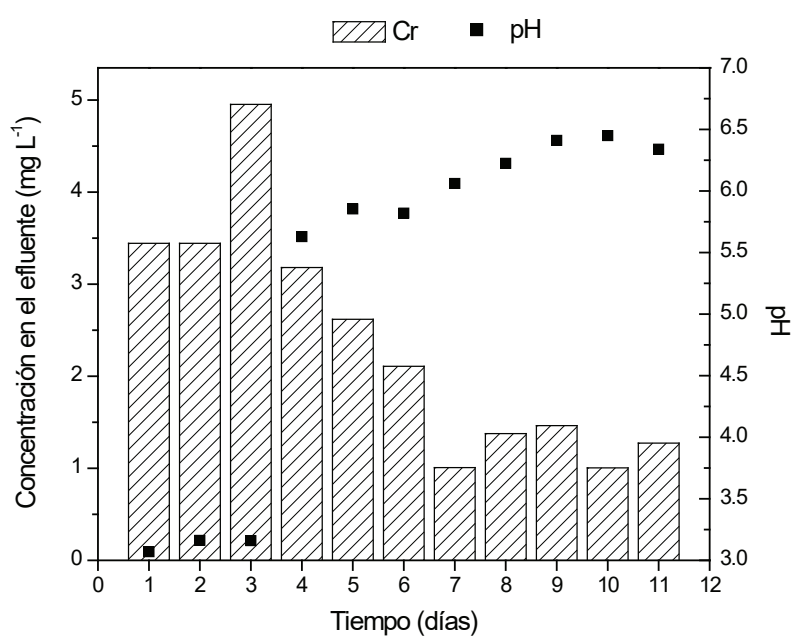

Figura 3. Comportamiento del pH y concentración de cromo en el efluente del humedal.

Figure 3. $\mathrm{pH}$ behavior and chromium concentration in the wetland effluent.

\section{Balance de masa para metales dentro del humedal}

La masa total de cada metal disponible para berro, se estimó con la masa de entrada menos el porcentaje precipitado de cada metal en el experimento control, el cual indicó un 80 y $50 \%$ de precipitación para $\mathrm{Pb}$ y $\mathrm{Cr}$, respectivamente. Los balances totales de masa para Pb (2 días) y Cr total (11 días) se muestran en las ecuaciones cinco y seis, respectivamente. Donde cada variable se encuentra relacionada con la fracción total de metal cuantificado.

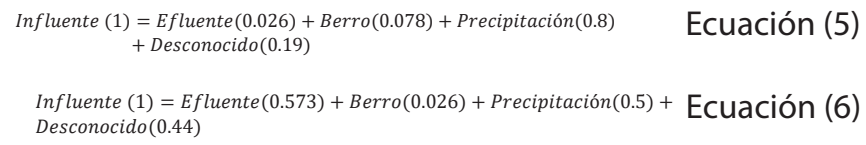

En ambas ecuaciones se observa que la fracción de metal acumulada en berro es baja comparada con el resto de las variables estimadas. Los fenómenos de precipitación generaron mayor retención del metal dentro del HCs y otros fenómenos fisicoquímicos y biológicos no evaluados en el presente estudio retuvieron el 44 y $19 \%$ de $\mathrm{Cr}$ total y $\mathrm{Pb}$, respectivamente. Algunos fenómenos no evaluados en el presente estudio pero que pudieron influir en el resultado, involucran la presencia de microorganismos anaerobios que generan condiciones reductoras en zonas anóxicas que modifican la biodisponibilidad de los metales en solución (Ye et al., 2013; Pedescoll et al., 2015), y la presencia de compuestos orgánicos e inorgánicos que están presentes en los ecosistemas que modifican la movilidad del metal (Azouzi et al., 2019), tal es el caso de los exudados radiculares (Ovecka y Takac, 2014).

Las simulaciones realizadas para conocer la especiación de $\mathrm{Pb}$ y $\mathrm{Cr}(\mathrm{VI})$ sin presencia de grava mostró formaciones de $\mathrm{Pb}\left(\mathrm{NO}_{3}\right)_{2}, \mathrm{PbNO}_{3}{ }^{+}$y $\mathrm{CrO}_{3} \mathrm{HPO}_{4}{ }^{2-}$ correspondientes al 58, 31 y $96 \%$, de la masa total precipitada de los metales, respectivamente. Mientras los productos formados en presencia de grava son $\mathrm{PbHCO}_{3}{ }^{+}$y $\mathrm{PbCO}_{3}$ para $\mathrm{Pb}$ que representan el $95 \%$ de la masa total precipitada. Así mismo, el $\mathrm{CaCrO}_{4}$ representa el $89 \%$ del Cr precipitado.

\section{CONCLUSIONES}

El berro tiene la capacidad de acumular $\mathrm{Cr}$ y $\mathrm{Pb}$ en raíces, considerándose una especie rizofiltradora y estabilizadora. Las interacciones químicas entre los metales influyen en su acumulación. Al aumentar las concentraciones individuales de cada metal en la solución, se incrementa su acumulación, pero disminuye el índice de tolerancia y el factor de bioconcentración para ambos metales. El factor de translocación aumenta a mayor concentración de cromo en solución. Las mezclas de metales favorecen la acumulación de un metal con respecto a otro e incrementan el índice de tolerancia del berro. La mayor remoción de los metales dentro del humedal fue causada por interacciones entre ambos metales y la grava. De acuerdo con el balance de masa realizado en el humedal artificial, se removió el 95 y >99.9\% de $\mathrm{Cr}(\mathrm{VI})$ y $\mathrm{Pb}$ de la solución. 


\section{REFERENCIAS}

Adriano, D.C. 2003. Trace Elements in Terrestrial Environments: Biogeochemistry, Bioavailability and Risks of Metals, Springer, New York.

APHA-AWWA-WEF. 2005. American Public Health Association, American Water Works Association Water Environment Federation, Standard Methods for the Examination of Water and Wasterwater, 21st Edition, Washington, D.C.

Aslan, M., Unlu, M.Y., Turkmen, N. y Yilmaz, Y.Z. 2003. Sorption of cadmium and effects on growth, protein content, and photosynthetic pigment composition of Nasturtium officinale R. Br. and Mentha aquatica L. B. Bulletin of Environmental Contamination and Toxicology. 71(2): 323329.

ATSDR, Agency for Toxic Substances and Disease Registry. Department of Health and Human Services. Draft toxicological profile for chromium; Draft Toxicological Profile for chromium. Consultado [5 febrero 2020] 2012. Disponible en: https://www.atsdr.cdc.gov/toxprofiles/tp7.pdf.

Azouzi, R., Charef, A., Ayed, L. y Khadhar, S. 2019. Effect of water quality on heavy metal redistribution and mobility in polluted agricultural soils in a semi-arid region. Pedosphere. 29(6): 730-739.

Cordeiro, C., Favas, P.J.C., Pratas, J., Sarkar, S.K. y Venkatachalam, P. 2016. Uranium accumulation in aquatic macrophytes in an uraniferous region: relevance to natural attenuation. Chemosphere. 156: 76-87.

Das, S., Gaswami, S. y Talukdar, A.D. 2014. A study on cadmium phytoremediation potential of water lettuce, Pistia stratiotes L. Bulletin Environmental Contamination Toxicology. 92(2): 169-174.

Devallois, V., Boyer, P., Boudenne, J.L. y Coulomb, B. 2008. Modelling the vertical profiles of $\mathrm{O}_{2}$ and $\mathrm{pH}$ in saturated freshwater sediments. International Journal of Limnology. 44(4): 275-288.

Duman, F., Leblebici, Z. y Aksoy A. 2009. Growth and bioaccumulation characteristics of watercress (Nasturtium officinale R. BR.) exposed to cadmium, cobalt and chromium. Chemical Speciation \& Bioavailability. 21(4): 257-265.

Duman F. y Ozturk K.F. 2010. Nickel accumulation and its effect on biomass, protein content and antioxidative enzymes in roots and leaves of watercress (Nasturtium officinale R. Br.). Journal of Environmental Sciences. 22: 526-532.

EPA. United States Environmental Protection Agency. Environmental topic, Lead regulations. Consultado [5 febrero 2020] 2018. Disponible en: https://www.epa.gov/ lead/lead-regulations

Favas, P.J.C., Pratas, J., y Prasad, M.N.V. 2012. Accumulation of arsenic bye aquatic plants in large-scale field conditions: opportunities for phytoremediation and bioindication. Science of the Total Environmen. 433: 390-397.

Favas, P.J.C., Pratas, J., Rodrigues, N., D’Souza, R., Varun M. y Paul, M.S. 2018. Metal (loid) accumulation in aquatic plants of a mining area: potential for water quality biomonitoring and biogeochemical prospecting. Chemosphere. 194: 158-170.

Frank, J.J., Poulakos, A.G., Tornero-Velez, R. y Xue, J. 2019. Systematic review and meta-analyses of lead (Pb) concentrations in environmental media (soil, dust, water, food, and air) reported in the United States from 1996 to 2016. Science of the Total Environment. 694: 133489.
Galal, T.M. y Shehata, H.S. 2015. Bioaccumulation and translocation of heavy metals by Plantago major L. grown in contaminated soils under the effect of traffic pollution. Ecological Indicators. 48: 244-251.

Hellerich, L.A., Nikolaidis, N.P. y Dobbs, G.M. 2008. Evaluation of the potential for the natural attenuation of hexavalent chromium within a sub-wetland ground water. Journal of Environmental Management. 88: 1513-1524.

Huang, K., Lin, L., Chen, F., Liao, M., Wang, J., Tang, Y., Lai, Y., Liang, D., Xia, H., Wang, X., y Ren, W. 2017. Effects of live Myriophyllum aquaticum and its straw on cadmium accumulation in Nasturtium officinale. Environmental Science and Pollution Research. 24: 22503-22509.

Islam, M.S., Saito, T. y Kurasaku, M. 2015. Phytofiltration of arsenic and cadmium by using an aquatic plant, Micranthemum umbrasoum: phytotoxicity, uptake kinetics, and mechanism. Ecotoxicology Environmental Safety. 112: 193-200.

Jasrotia, S., Kansal, A. y Mehra, A. 2017. Performance of aquatic plants species for phytoremediation of arseniccontaminated water. Applied Water Science. 7: 889-896.

Jin, R., Liu, Y., Liu, G., Tian, T., Qiao, S. y Zhou, J. 2017. Characterization of product and potential mechanism of $\mathrm{Cr}$ (VI) reduction by anaerobic activated sludge in a sequencing batch reactor. Scientific Reports. 7(1): 1681.

Kara, Y. 2005. Bioaccumulation of $\mathrm{Cu}, \mathrm{Zn}$ and $\mathrm{Ni}$ from the wastewater by treated Nasturtium officinale. International Journal of Environmental Science and Technology. 2: 63-67.

Keser, G. y Saygideger, S. 2010. Effects of lead on the activities of antioxidant enzymes in watercress, Nasturtium officinale R. Br. Biological Trace Element Research. 137: 235-243.

Klimek-Szczykutowicz, M., Szopa, A. y Ekiert, H. 2018. Chemical composition, traditional and professional use in medicine, application in environmental protection, position in food and cosmetics industries, and biotechnological studies of Nasturtium officinale (watercress). A review. Fitoterapia. 129: 283-292.

Li, K., Lin, L., Wang, J., Xia, H., Liang, D., Wang, X., Liao, M., Wang, L., Liu, L., Chen, C. y Tang, Y. 2017. Hyperaccumulator straw improves the cadmium phytoextraction efficiency of emergent plant Nasturtium officinale. Environmental Monitoring and Assessment. 189: 374.

Lima, L., Olivares-Rieumont, S., Columbie, I., de la Rosa-Menderos, D. y Gil-Castillo, R. 2005. Niveles de plomo, zinc, cadmio y cobre en el Rio Almendares, Ciudad Habana, Cuba. Revista Internacional de Contaminación Ambiental. 21(3): 115-124.

Lin, L., Luo, L., Lian, M., Zhang, X. y Yang, D. 2015. Cadmium accumulation characteristics of emerged plant Nasturtium officinale R.BR. Resources and Environment in the Yangtze Basin. 4: 50-60.

Liu, X., Gao, Y., Khan, S., Duan, G., Chen, A., Ling, L., Zhao, L., Liu, Z. y Wu, X. 2008. Accumulation of $\mathrm{Pb}, \mathrm{Cu}$, and $\mathrm{Zn}$ in native plants growing on contaminated sites and their potential accumulation capacity in Heqing, Yunnan. Journal of Environmental Sciences. 20(12): 1469-1474.

Liu, L., Wang, S., Guo, X. y Wang, H. 2019. Comparison of the effects of different maturity composts on soil nutrient, plant growth and heavy metal mobility in the contaminated soil. Journal of Environmental Management. 250:109525.

López-Luna, J., González-Chávez, M.C., Esparza-García, F.J. y Rodríguez-Vázquez, R. 2009. Toxicity assessment of soil amended with tannery sludge, trivalent chromium and 
hexavalente chromium, using wheat, oat and sorghum plants. Journal of Hazardous Materials. 163(2-3): 829-834.

Maceda-Veiga, A., Monroy, M., Navarro, E., Viscor, G. y de Sostoa, A. 2013. Metal concentrations and pathological responses of wild native fish exposed to sewage discharge in a Mediterranean river. Science of the Total Environment. 449: 9-19.

Marchand, L., Mench, M., Jacob, D.L. y Otte, M.L. 2010. Metal and metalloid removal in constructed wetlands, with emphasis on the importance of plants and standardized measurements: A review. Environmental Pollution. 158(12): 3447-3461.

Naiya, T.K., Bhattacharya, A.K., Mandal, S. y Das, S.K. 2009. The sorption of lead (II) ions on rice husk ash. Journal of Hazardous Materials.163: 1254-1264.

Ng, C.C., Rahman, M.M., Boyce, A.N. y Abas, M.R. 2016. Heavy metals phyto-assessment in commonly grown vegetables: water spinach (I. aquatic) and okra (A. esculentus). Springer Plus. (5) 1: 469-477.

Olmos-Márquez, M.A., Alarcón-Herrera, M.T. y MartínDomínguez, I.R. 2012. Performance of Eleocharis macrostachya and its importance for arsenic retention in constructed wetlands. Environmental Science and Pollution Research. 19(3): 763-771.

Ovecka, M. y Takac, T. 2014. Managing heavy metal toxicity stress in plants: Biological and biotechnological tools. Biotechnology Advances. 32: 73-86.

Ozturk, F., Duman, F., Leblebici, Z. y Temizgul R. 2010. Arsenic accumulation and biological responses of watercress (Nasturtium officinale $R$. Br.) exposed to arsenite. Environmental and Experimental Botany. 69(2): 167-174.

Pawlisz, A.V., Kent, R.A., Schneider, U.A y Jefferson, C. 1997. Canadian water quality guidelines for chromium. Environmental Toxicology and Water Quality.1282: 123-183.

Pedescoll, A., Sidrach-Cardona, R., Hijosa-Valsero, M. y Bécares E. 2015. Design parameters affecting metals removal in horizontal constructed wetlands for domestic wastewater treatment. Ecological Engineering. 80: 92-99.

Picco, P., Hasuoka, P., Verni, E., Savio, M. y Pacheco, P. 2019. Arsenic species uptake and translocation in Elodea canadensis. International Journal of Phytoremediation. 21(7): 693-698.

Rascio, N. y Naviri-Izzo F. 2011. Heavy metal hyperaccumulating plants: how and why do they do it? And what makes them so interesting?. Plant Science. 180(2): 169-181.

Ribeiro de Souza, S.R., López de Andrade, S.A., Anjos de Souza, L. y Aparecida, M. 2012. Lead tolerance and phytoremediation potential of Brazilian leguminous tree species at the seedling stage. Journal of Environmental Management. 110: 299-307.

Rose, P., Huang, Q., Ong, C.N. y Whiteman, M. 2005. Broccoli and watercress suppress matrix metalloproteinase- 9 activity and invasiveness of human MDA-MB-231 breast cancer cells. Toxicology and Applied Pharmacology. 209(2): 105-113.
Saygideger, S. y Dogan, M. 2005. Influence of pH on lead uptake, chlorophyll and nitrogen content of Nasturtium officinale $R$. $B r$. and Mentha aquatica L. Journal of Environmental Biology. 26(4): 753-759.

Seth, C.S. 2011. A review on mechanisms of plant tolerance and role of transgenic plants in environmental clean-up. Botanical Review. 78(1): 32-62.

Shi, X., Zhang, X., Chen, G., Chen, Y., Wang, L. y Shan, X. 2011. Seedling growth and metal accumulation of selected woody species in copper and lead/zinc mine tailings. Journal of Environmental Sciences. 23(2): 266-274.

Shri, M., Kumar, S., Chakrabarty, D., Trivedi, P. K., Mallick, S., Misra, P., Shukla, D., Mishra, S., Srivastava, S., Tripathi, R.D. y Tuli, R. 2009. Effect of arsenic on growth oxidative stress, and antioxidant system in rice seedlings. Ecotoxicology Environmental Safety. 72(4): 1102-1110.

Souza, T.D., Borges, A. C., Matos, A.T., Veloso, R.W. y Braga, A.F. 2018. Kinetics of arsenic absorption bye the species Eichhornia crassipes and Lemna valdiviana under optimized conditions. Chemosphere. 209: 866-874.

Vidal de Campos, F., Alves de Oliveira, J., Alves da Silva, A., Ribeiro, C. y dos Santos F. 2019. Phytoremediation of arsenite-contaminated environments: is Pistia stratiotes $L$. a useful tool?. Ecological Indicators. 104: 794-801.

Wang, Z., Liu, X. y Qin, H. 2019. Bioconcentration and translocation of heavy metals in the soil-plants system in Machangqing copper mine, Yunnan Province, China. Journal of Geochemical Exploration. 200: 159-166.

Yadav, A.K., Abbassi, R., Kumar, N., Satya, S., Sreekrishnan, T.R. y Mishra, B.K. 2012. The removal of heavy metals in wetland microcosms: Effects of bed depth, plant species, and metal mobility. Chemical Engineering Journal. 211-212: 501-507.

Yadav, A.K., Kumar, N., Sreekrishnan, T.R., Santosh, S. y Bishnoi, N.R. 2010. Removal of chromium and nickel from aqueous solution in constructed wetland: Mass balance, adsorptiondesorption and FTIR study. Chemical Engineering Journal. 160: 122-128.

Ye, S., Laws, E.A.y Gambrell, R. 2013. Trace element remobilization following the resuspension of sediments under controlled redox conditions: City Park Lake, Baton Rouge, LA. Applied Geochemistry. 28: 91-99.

Zurayk, R., Sukkariyah, B., y Baalbaki, R. 2001a. Common hydrophytes as bioindicators of nickel, chromium and cadmium pollution. Water Air and Soil Pollution. 127: 373388.

Zurayk, R., Sukkariyah, B., Baalbaki R. y Ghanem, D.A. 2001b. Chromium phytoaccumulation from Solution by Selected Hydrophytes. International Journal of Phytoremediation. 3: 335-350. 